\title{
Mother-to-Children Plasmodium falciparum Asymptomatic Malaria Transmission at Saint Camille Medical Centre in Ouagadougou, Burkina Faso
}

\author{
Zoenabo Douamba, ${ }^{1,2}$ Nangnéré Ginette Laure Dao, ${ }^{2,3}$ \\ Théodora Mahoukédé Zohoncon, ${ }^{1,2,4}$ Cyrille Bisseye, ${ }^{2,5}$ \\ Tegwindé Rebeca Compaoré, ${ }^{1,2}$ Jacques Gilbert Kafando, ${ }^{2}$ Bavouma Charles Sombie, \\ Djeneba Ouermi, ${ }^{1,2,4}$ Florencia W. Djigma, ${ }^{1,2,4}$ Paul Ouedraogo, ${ }^{2,4}$ Nadine Ghilat, ${ }^{4}$ \\ Virginio Pietra, ${ }^{2,4}$ Vittorio Colizzi, ${ }^{2,6}$ and Jacques Simpore ${ }^{1,2,3,4}$ \\ ${ }^{1}$ Laboratory of Molecular Biology and Genetics (LABIOGENE), University of Ouagadougou, 03 P.O. Box 7021, \\ Ouagadougou 03, Burkina Faso \\ ${ }_{2}^{2}$ Pietro Annigoni Biomolecular Research Centre (CERBA), 01 P.O. Box 364, Ouagadougou 01, Burkina Faso \\ ${ }^{3}$ University St. Thomas Aquinas of Ouagadougou, 06 P.O. Box 10212, Ouagadougou 06, Burkina Faso \\ ${ }^{4}$ Saint Camille Medical Centre of Ouagadougou, 01 P.O Box 364, Ouagadougou 01, Burkina Faso \\ ${ }^{5}$ Laboratory of Molecular and Cellular Biology, University of Science and Technique of Masuku (USTM), P.O. Box 943, \\ Franceville, Gabon \\ ${ }^{6}$ Department of Biology, UNESCO Chair in Biotechnology, Laboratory of Immunology \& Pathology, University of Rome Tor Vergata, \\ Scientific Research Avenue snc, 00143 Rome, Italy
}

Correspondence should be addressed to Jacques Simpore; jacques.simpore@yahoo.fr

Received 8 September 2014; Accepted 1 November 2014; Published 23 November 2014

Academic Editor: Polrat Wilairatana

Copyright (C) 2014 Zoenabo Douamba et al. This is an open access article distributed under the Creative Commons Attribution License, which permits unrestricted use, distribution, and reproduction in any medium, provided the original work is properly cited.

Background. Malaria's prevalence during pregnancy varies widely in parts of sub-Saharan Africa, including Burkina Faso. The objective of this study was to evaluate the incidence of mother-to-child malaria transmission during childbirth at St. Camille Medical Centre in the city of Ouagadougou. Methods. Two hundred and thirty-eight (238) women and their newborns were included in the study. Women consenting to participate in this study responded to a questionnaire that identified their demographic characteristics. Asymptomatic malaria infection was assessed by rapid detection test Acon (Acon Malaria Pf, San Diego, USA) and by microscopic examination of Giemsa-stained thick and thin smears from peripheral, placental, and umbilical cord blood. Birth weights were recorded and the biological analyses of mothers and newborns' blood were also performed. Results. The utilization of long-lasting insecticidal nets (LLINs) and intermittent preventive treatment with sulfadoxine-pyrimethamine (SP) were $86.6 \%$ and $84.4 \%$, respectively. The parasitic infection rates of $9.5 \%, 8.9 \%$, and $2.8 \%$ were recorded, respectively, for the peripheral, placental, and umbilical cord blood. Placental infection was strongly associated with the presence of parasites in the maternal peripheral blood and a parasite density of $>1000$ parasites $/ \mu \mathrm{L}$. Conclusion. The prevalence of congenital malaria was reduced but was associated with a high rate of mother-to-child malaria transmission.

\section{Introduction}

In sub-Saharan Africa, about thirty-two million pregnant women are living in areas at risk for Plasmodium falciparum malaria transmission [1]. The prevalence of gestational malaria is highly variable in these regions. Indeed, prevalence of $5 \%, 28 \%$, and $68.3 \%$ was reported, respectively, in Ghana [2], Uganda [3], and Nigeria [4]. Two previous studies in Burkina Faso showed that the prevalence of malaria among pregnant women ranged from $28.2 \%$ to $42.7 \%$ [5, 6]. Malaria 
infection during pregnancy is potentially dangerous because of the risk for maternal and infantile health [7]. Even asymptomatic, Plasmodium falciparum caused malaria is responsible for anemia in pregnant women $[6,8]$. The severity of malaria in pregnant women is due to placental sequestration of infected red blood cells $[9,10]$. This sequestration alters placental function [11] and causes nutrient deficiencies that result in abortions, intrauterine growth retardation, stillbirth, and premature birth $[12,13]$. Gestational malaria is responsible for a high maternal and infantile morbidity and is causing about 75000 to 200000 deaths of infants each year [14]. Malaria vertical transmission could have serious consequences for the newborn such as a high susceptibility to malaria infection during the first months of life [15, 16] and also an early susceptibility to other infections [17, 18]. This susceptibility to infection is due to neonatal $\mathrm{T}$ cells imbalance and proinflammatory and anti-inflammatory immune responses after their sensitization by Plasmodium falciparum in utero [19-21]. Placenta malaria infection is high in Burkina Faso and it is characterized by a low rate of neonatal infection [5]. However, few studies have investigated malaria vertical transmission and its impact on the newborns' health. The objectives of this study were (i) to assess the coverage of malaria prophylaxis with intermittent preventive treatment (IPT) based on sulfadoxine-pyrimethamine (SP) in parturients, (ii) to determine the prevalence of asymptomatic malaria in parturients, and (iii) to estimate the prevalence of placental malaria in asymptomatic pregnant women and the incidence of congenital malaria among their newborns.

\section{Materials and Methods}

2.1. Study Population. This cross-sectional study was conducted from September 2013 to February 2014 at Saint Camille Medical Center (CMSC) of Ouagadougou. Ouagadougou is located in the center of Burkina Faso with over a million and a half inhabitants. The climate in the area is characteristic of the Sudanese savannah, with a rainy season from June to October, the cold and dry season from November to January, and the hot and dry season from February to May. The transmission of malaria is stable with a high seasonal transmission from June to November. Pregnant women without clinical signs of malaria and who gave birth were enrolled in this study. Symptoms of clinical suspicion of malaria include fever, headache, vomiting, nausea, abdominal pain, diarrhea, and myalgia. Since malaria symptoms can be confused with clinical manifestations occurring in pregnant women during labor and delivery, only nonfebrile pregnant women were considered asymptomatic for malaria. Only 238 women who consented to participate in the study were recruited. These women completed a questionnaire to determine their demographic characteristics and the malaria prophylaxis used.

2.2. Blood Collection. From the women who consented to participate in the study, venous, placental, and umbilical cord blood was collected in an EDTA impregnated tube for hematological tests or dry tube for biochemical analysis.
2.3. Biological Analysis. Asymptomatic malaria infection was assessed by rapid detection test (RDT) Acon (Acon Malaria Pf, San Diego, USA) and by microscopic examination of Giemsa-stained thick and thin smears from peripheral, placental, and umbilical cord blood. All slides were read twice by experienced microscopists and the discrepancies resolved by a third reader (limit of detection, approximately 2 parasites $/ \mathrm{mL}$ ). We used the standard method to determine parasitaemia as in most studies conducted in Africa. For thick films, parasites and leukocytes were counted in the same fields until 200 or 500 leukocytes were counted. Parasite densities were estimated using an assumed leukocyte count of 8,000 leukocytes $/ \mu \mathrm{L}$. Parasite densities were calculated according to the following formula: number of parasites counted $\times 8000 /$ number of leucocytes counted $[5,22]$. Haemoglobin level test was performed using a commercial colorimetric kit (Cypress Diagnostics, Langdorp, Belgium) following the manufacturer's instructions. The determination of aspartate aminotransferase (ASAT), alanine aminotransferase (ALAT), and creatinine levels was performed using commercial kits in a Cobas machine (Roche Diagnostics $\mathrm{GmbH}$, Sandhofer Strasse).

2.4. Statistical Analysis. Data were analyzed using Statistical Package for Social Sciences (SPSS version 20; SPSS Inc., Chicago, IL, USA) and EPI-Info version $6.04 \mathrm{dfr}$ (CDC, Atlanta, GA, USA). $P$ values below 0.05 were considered statistically significant.

\section{Results}

3.1. Parturients' Sociodemographic Characteristics. A total of 238 parturients aged from 17 to 42 years were included in the study. The mean age of women was $26.46 \pm 4.96$ years. The age group of 21 to 25 years old was the most represented. The majority of women (86.6\%) used long-lasting insecticidal net (LLIN) for vector control. Most women took at least one dose of sulfadoxine-pyrimethamine (SP) as an intermittent preventive treatment against malaria. Anemia was diagnosed in $18.4 \%$ of pregnant women, and $3.4 \%(8 / 238)$ had severe anemia (Table 1).

3.2. Clinical Characteristics of the Newborns. Two hundred and thirty-eight (238) mother-infant pairs were included in the study. Newborns had a mean weight of $3157 \pm 394$ grams (2400-4430 g). A small number of children $(1.7 \%)$ were less than $2500 \mathrm{~g}$ birth weight. The mean of hemoglobin level in newborns rate was $14.9 \mathrm{~g} / \mathrm{dL}$. Anemia was present in $10.1 \%$ of neonates. Over $90 \%$ of the infants included in our study had an elevated creatinine. Transaminases were also measured in newborns and over $84 \%$ of them had normal aminotransferase levels (Table 2).

No association was found between the malaria infection in parturients and placental and umbilical cord blood and the clinical parameters of newborns.

3.3. Prevalence of Asymptomatic Malaria in Pregnant Women. The parasites were present in the peripheral blood of $8.8 \%$ (21/238) of the pregnant women. 
TABLE 1: Characteristics of parturients.

\begin{tabular}{lc}
\hline Characteristics & Number $(\%)$ \\
\hline Age (years) & $15(6.30)$ \\
$\leq 20$ & $101(42.44)$ \\
$21-25$ & $76(31.93)$ \\
$26-30$ & $46(19.33)$ \\
$31-39$ & \\
Hemoglobin level (g/dL) & $8(3.36)$ \\
$<7$ (severe anaemia) & $13(5.46)$ \\
$7-9.9$ (moderate anaemia) & $25(10.50)$ \\
$10-10.9$ (slight anaemia) & $192(80.67)$ \\
$\geq 11$ (no anaemia) & \\
Number of SP intakes & $37(15.55)$ \\
0 & $55(23.11)$ \\
1 & $145(60.92)$ \\
2 & $1(0.42)$ \\
3 & \\
Long-lasting insecticidal net (LLIN) & $206(86.55)$ \\
Yes & $32(13.45)$ \\
No &
\end{tabular}

TABLE 2: Characteristics of newborns.

\begin{tabular}{lc}
\hline Characteristics & Number $(\%)$ \\
\hline Weight (gram) & \\
$\quad<2500$ (low) & $234(98.68)$ \\
$\quad \geq 2500$ (normal) & \\
Hemoglobin level (g/dL) & $24(10.06)$ \\
$\quad<12.5$ (anaemia) & $214(89.92)$ \\
$\quad>12.5$ (no anaemia) & \\
Creatinemia $(\mu \mathrm{mol} / \mathrm{L})$ & $223(93.70)$ \\
53 to 106 (normal) & $13(6.30)$ \\
$>106($ elevated) & \\
Transaminases GOT (U/L) & 0 \\
$\quad<15$ (low) & $226(94.96)$ \\
15 to 60 (normal) & $12(5.04)$ \\
$>60$ (elevated) & \\
Transaminases GPT (U/L) & 0 \\
$\quad<5$ (low) & $202(84.87)$ \\
5 to 25 (normal) & $36(15.13)$ \\
$\quad>25$ (elevated)
\end{tabular}

P. falciparum was present in all cases of infections. The plasmodial mixed infections were found in 6 of the 21 mothers diagnosed positive by RDT. Thick smears revealed the presence of $P$. malariae in 2 mixed infections and $P$. ovale in one case. The geometric mean of parasites in the parturients was 14,477 parasites $/ \mu \mathrm{L}$ (118-115073 parasites $/ \mu \mathrm{L}$ ) (Table 3). The infection rate $(12.4 \%)$ was higher during the period of September to December compared to the one of January to February (8.2\%), but the difference was not significant.
3.4. Prevalence of Placental Malaria. The microscopy revealed the presence of parasites in placental blood, 7.1\% (17/238) of the specimens tested. RDT also showed the presence of 6 mixed plasmodial infections in placental blood, including 2 cases of $P$. malariae. The geometric mean of the parasitaemia observed was 21,630 parasites/ $\mu \mathrm{L}$ (ranged 143112,396 parasites $/ \mu \mathrm{L}$ ) (Table 3). The placental infection rate was also higher from the period of September to December (9.0\%) compared to the one of January to February (1.6\%).

3.5. Incidence of Congenital Malaria. We diagnosed 27 maternal and placental malaria and 5 of 238 umbilical cord blood infections. The incidence of congenital malaria was $2.1 \%$ and the prevalence of mother-to-child asymptomatic malaria transmission was $18.5 \%$. Plasmodium falciparum was the only parasite species present in the umbilical cord blood. The parasite density was low in the cord blood compared to those observed in placental and peripheral blood with a mean of 1444 parasites $/ \mu \mathrm{L}$ (231-5102 parasites $/ \mu \mathrm{L}$ ) (Table 3 ).

3.6. Association between Peripheral, Placental, and Umbilical Cord Malaria Parasites and LLINs and Intermittent Preventive Treatment with SP. As shown in Table 4, pregnancyassociated malaria was not associated with the use of LLINs, neither with intermittent preventive treatment with SP (IPT-SP). Of the 17 positive thick placental blood smears, the majority (11/17) was from women with positive thick peripheral blood smears (Table 5). Positive thick cord blood smears were from women with positive thick peripheral blood smears and those with both peripheral and placental parasitaemia. Among the women with both peripheral and placental malaria parasites, the majority of placental infection (9/11) was observed among women with a mean of parasite density superior ( $>$ ) to 1000 parasites $/ \mu \mathrm{L}$ (Table 5 ).

\section{Discussion}

In areas with moderate or high $P$. falciparum malaria endemicity, WHO recommends the use of LLINs and intermittent preventive treatment with SP (IPT-SP) for pregnant women in order to prevent malaria and its effects during pregnancy. IPT with at least two doses of sulfadoxinepyrimethamine administered during the second and third trimester of pregnancy is an alternative prevention strategy whose effectiveness has been demonstrated by several studies in Burkina Faso and elsewhere in sub-Saharan Africa [23, 24] by reducing the rate of maternal and placental Plasmodium falciparum infestation. Most women of our study received two doses of SP in the second and third trimester of their pregnancies. Those who received one dose came in their third trimester.

In this study, the use of malaria preventive measures such as LLINs and IPT-SP was high: $86.6 \%$ and $84.5 \%$, respectively. We observed an increase rate in the use of LLINs and IPT compared to the rates of $42 \%$ and $27 \%$ reported in 2012 in the same health center [8]. This could be explained by the positive impact of awareness campaigns among pregnant women on the use of LLINs and their wide distribution in the population 
TABLE 3: Malaria parasites detected in peripheral, placental, and umbilical cord blood among parturients.

\begin{tabular}{|c|c|c|c|c|c|c|}
\hline \multirow[b]{2}{*}{ Samples } & \multicolumn{2}{|c|}{ Thick peripheral blood smears } & \multicolumn{2}{|c|}{ Thick placental blood smears } & \multicolumn{2}{|c|}{ Thick cord blood smears } \\
\hline & $\begin{array}{c}\text { Parasite density } \\
\text { (parasites } / \mu \mathrm{L} \text { ) }\end{array}$ & $\begin{array}{c}\text { Type of } \\
\text { plasmodium } \\
\text { parasite }\end{array}$ & $\begin{array}{l}\text { Parasite density } \\
\text { (parasites } / \mu \mathrm{L})\end{array}$ & $\begin{array}{c}\text { Type of } \\
\text { plasmodium } \\
\text { parasite }\end{array}$ & $\begin{array}{c}\text { Parasite density } \\
\text { (parasites } / \mu \mathrm{L} \text { ) }\end{array}$ & $\begin{array}{c}\text { Type of } \\
\text { plasmodium } \\
\text { parasite }\end{array}$ \\
\hline M4 & & & 143 & P.f. & & \\
\hline M6 & 8,157 & P. $f$. & & & & \\
\hline M16 & 390 & P. $f$. & & & & \\
\hline M38 & 14,811 & P. $f$. & 12,256 & P. $f$. & & \\
\hline M49 & 11,647 & P.f. & 12,317 & P. $f$. & 5,102 & P. f. \\
\hline M66 & 11,647 & $\begin{array}{l}\text { P.f. and } \\
\text { P. } m \text {. }\end{array}$ & 522 & P. $f$. & 231 & P. f. \\
\hline M90 & & & 175 & P. f. & & \\
\hline M92 & 1,534 & P. $f$. & & & 512 & P. $f$. \\
\hline M95 & 270 & P. $f$. & 509 & $\begin{array}{l}\text { P.f. and } \\
\text { P. m. }\end{array}$ & & \\
\hline M114 & 13,044 & P. $f$. & & & & \\
\hline M126 & 118 & P. $f$. & 716 & P. $f$. & 517 & P. $f$. \\
\hline M131 & 135 & P. $f$. & & & & \\
\hline M133 & 827 & P. $f$. & & & & \\
\hline M147 & 26,862 & P.f. & 16,196 & P.f. & & \\
\hline M155 & 1,450 & P. f. and P. $m$. & 588 & P. f. and P. $m$. & & \\
\hline M160 & & & 2,470 & P.f. & & \\
\hline M162 & & & 2,263 & P. f. & & \\
\hline M167 & 115,073 & P. $f$. & 112,396 & P. $f$. & & \\
\hline M169 & & & 18,600 & P. $f$. & & \\
\hline M173 & 16,985 & P. $f$. & 82,325 & P. $f$. & & \\
\hline M175 & 61,635 & P. $f$. & 99,445 & P. $f$. & & \\
\hline M177 & 4,610 & $\begin{array}{l}P . f . \text { and } \\
\text { P. o. }\end{array}$ & 1,064 & P. f. & & \\
\hline M186 & 2,370 & P. $f$. & & & & \\
\hline M195 & 2,857 & P. $f$. & & & & \\
\hline M196 & 2,105 & P. $f$. & & & 857 & P. $f$. \\
\hline M199 & 1,200 & P. $f$. & & & & \\
\hline M200 & & & 5,720 & P.f. & & \\
\hline
\end{tabular}

P. f: Plasmodium falciparum; P. m.: Plasmodium malariae; P. o.: Plasmodium ovale. Parasite densities presented are from P. falciparum.

TABLE 4: Association between intermittent preventive treatment and use of LLIN and pregnancy-associated malaria.

\begin{tabular}{lccc}
\hline \multirow{2}{*}{ Parameters } & \multicolumn{2}{c}{ Thick peripheral blood smears } & \multirow{2}{*}{ N value } \\
& Pegative & Positive & \\
\hline IPT with SP & & & \\
No & $34 / 37(91.9 \%)$ & $3 / 37(8.1 \%)$ & NS \\
Yes & $181 / 201(90.1 \%)$ & $20 / 201(9.9 \%)$ & \\
LLINs & & & \\
No & $28 / 32(87.5 \%)$ & $4 / 32(12.5 \%)$ & NS \\
Yes & $187 / 206(90.8 \%)$ & $19 / 206(9.2 \%)$ & \\
\hline
\end{tabular}

NS: not significant.

by the Ministry of Health. Our results are similar to those observed in 2013 in the two major cities of Burkina Faso [25].
However, this rate is higher than that reported recently in Ghana [2].

The mean birth weight in the present study is comparable to that reported in a previous study in Burkina Faso [5]. We found a prevalence of $1.7 \%$ of low birth weight. This prevalence is much lower than that of $15.8 \%$ reported by Ouédraogo et al. [5] but is comparable to the prevalence of 3.3\% reported recently in Ghana [2]. The prevalence of anemia among newborns was of $10.1 \%$. This rate is lower than those reported in two previous studies in Malawi (23.4\%) and Ghana (40\%) [26, 27]. This difference could be explained by the high prevalence of anemia among parturients and by the presence of placental and neonatal malaria in their study populations. A parasite infection rate of $8.8 \%$ was found in the peripheral blood of pregnant women. It is less than $28 \%$ and $68.3 \%$, respectively, reported in Uganda [3] and Nigeria 
TABLE 5: Association between maternal malaria infection and IPT-SP and placental and neonatal malaria.

\begin{tabular}{|c|c|c|c|c|c|c|}
\hline \multirow{2}{*}{ Parameters } & \multicolumn{2}{|c|}{ Thick placenta blood smears } & \multirow{2}{*}{$P$ value } & \multicolumn{2}{|c|}{ Thick cord blood films } & \multirow{2}{*}{$P$ value } \\
\hline & Negative & Positive & & Negative & Positive & \\
\hline \multicolumn{7}{|c|}{ Thick peripheral blood films } \\
\hline Negative & $\begin{array}{c}209 / 217 \\
96.3 \%\end{array}$ & $\begin{array}{l}8 / 217 \\
3.7 \%\end{array}$ & \multirow{2}{*}{$<0.001$} & $\begin{array}{r}216 / 217 \\
99.5 \%\end{array}$ & $\begin{array}{l}1 / 217 \\
0.5 \%\end{array}$ & \multirow{2}{*}{ NA } \\
\hline Positive & $\begin{array}{l}12 / 21 \\
57.1 \%\end{array}$ & $\begin{array}{c}9 / 21 \\
42.9 \%\end{array}$ & & $\begin{array}{c}16 / 21 \\
76.2 \%\end{array}$ & $\begin{array}{c}5 / 21 \\
23.8 \%\end{array}$ & \\
\hline \multicolumn{7}{|l|}{ IPT-SP } \\
\hline No & $\begin{array}{l}34 / 37 \\
91.9 \%\end{array}$ & $\begin{array}{l}3 / 37 \\
8.1 \%\end{array}$ & \multirow{2}{*}{ NS } & $\begin{array}{l}36 / 37 \\
97.3 \%\end{array}$ & $\begin{array}{l}1 / 37 \\
2.7 \%\end{array}$ & \multirow{2}{*}{ NA } \\
\hline Yes & $\begin{array}{c}182 / 201 \\
90.5 \%\end{array}$ & $\begin{array}{c}19 / 201 \\
9.5 \%\end{array}$ & & $\begin{array}{c}196 / 201 \\
97.5 \%\end{array}$ & $\begin{array}{l}5 / 201 \\
2.5 \%\end{array}$ & \\
\hline \multicolumn{7}{|c|}{ Parasite density } \\
\hline$<1000$ & $\begin{array}{c}3 / 11 \\
27.27 \%\end{array}$ & $\begin{array}{c}8 / 11 \\
72.73 \%\end{array}$ & \multirow{2}{*}{ NS } & $\begin{array}{l}0 / 5 \\
0 \%\end{array}$ & $\begin{array}{c}1 / 5 \\
20 \%\end{array}$ & \multirow{2}{*}{ NA } \\
\hline$\geq 1000$ & $\begin{array}{c}7 / 16 \\
43.8 \% \\
\end{array}$ & $\begin{array}{c}9 / 16 \\
56.2 \%\end{array}$ & & $\begin{array}{l}0 / 5 \\
0 \% \\
\end{array}$ & $\begin{array}{c}4 / 5 \\
80 \%\end{array}$ & \\
\hline
\end{tabular}

NS: not significant; NA: not applicable.

[4]. This difference could be due to the high IPT-SP adherence rate in Burkina Faso [28] and the high prevalence of malaria among pregnant women in some parts of Nigeria [29-31]. Malaria transmission is higher in Burkina Faso, from the period of July to December, during the rainy season. The parasite infection rate in parturients was higher during the period of September to December.

In this study, Plasmodium malariae was identified in 11.1\% of asymptomatic malaria cases in pregnant women. Our results are consistent with those of a previous study among children which had reported the prevalence of $P$. malariae ranging from 0.9 to $13.2 \%$ in Burkina Faso [32]. The prevalence of $P$. malariae reported in this study is higher than the prevalence of $3.6 \%$ found in Nigeria in pregnant women [33]. Despite the low prevalence of $P$. ovale in Burkina Faso, which varies from 0.5 to 1.8 [32], we identified $3.7 \%$ of $P$. ovale asymptomatic cases in parturients in this study. This is consistent with a recent study which has shown that $P$. ovale and $P$. malariae represent $5.9 \%$ of malaria infections in Senegal [34]. The prevalence of placental malaria was $7.1 \%$ among the women. This prevalence is lower than the prevalence of $19.5 \%$ and $55.2 \%$ reported in Burkina Faso [5] and Nigeria [31], respectively. Moreover, the placental malaria prevalence is elevated than that reported in a recent study in Ghana [2]. The difference found could be explained by an intraregional variation in the prevalence of placental and neonatal malaria $[5,30]$. The malaria infection rate of umbilical cord blood was $2.1 \%$. This rate is comparable to the rate of $1.4 \%$ reported in two previous studies in Burkina Faso [5] and India [35].

There is a strong association between maternal parasite density, placental malaria, and cord blood parasitaemia. This association was also shown in Burkina Faso $[5,36]$.
However, we did not find an association between maternal and placental malaria infection and umbilical cord parasitaemia. This could be explained by the limited number of cases of congenital malaria in our study.

Our study has some limitations such as the sample size but also the threshold of parasites detection related to standard microscopy. In fact, the latter may underestimate the number of asymptomatic malaria cases among parturients and placental and cord blood malaria.

\section{Conclusion}

The high use of preventive measures such as LLINs and IPT-SP among parturients has resulted in the reduction of asymptomatic malaria and severe anemia. These efforts should be pursued because there is a high risk of mother-tochild asymptomatic malaria transmission.

Asymptomatic malaria in women is underestimated because placental malaria can be observed in parturients in the absence of parasites in their peripheral blood.

\section{Ethical Approval}

The Saint Camille Medical Center Ethics committee approved this study and each woman has given written consent before blood collection. All subjects infected with malaria (mother and children) were treated according to the national protocol in place.

\section{Conflict of Interests}

The authors declare that there is no conflict of interests regarding the publication of this paper. 


\section{Acknowledgments}

The authors are grateful to all the participants of this study. They would like to thank the Italian Episcopal Conference (CEI) and the West African Economic and Monetary Union (WAEMU) through the Programme d'Appui et de Développement des Centres d'Excellence Régionaux (PACER II) for their financial support. The authors are grateful to all the pregnant women and the Saint Camille Medical staff who have participated in this study. The authors also would like to thank all the staff of CERBA/LABIOGENE for their help.

\section{References}

[1] S. Dellicour, A. J. Tatem, C. A. Guerra, R. W. Snow, and F. O. Ter Kuile, "Quantifying the number of pregnancies at risk of malaria in 2007: a demographic study," PLoS Medicine, vol. 7, no. 1, Article ID e1000221, 2010.

[2] J. K. Stephens, M. F. Ofori, I. A. Quakyi, M. L. Wilson, and B. D. Akanmori, "Prevalence of peripheral blood parasitaemia, anaemia and low birthweight among pregnant women in a suburban area in coastal Ghana," Pan African Medicine Journal, vol. 17, supplement 1, p. 3, 2014.

[3] P. de Beaudrap, E. Turyakira, L. J. White et al., "Impact of malaria during pregnancy on pregnancy outcomes in a Ugandan prospective cohort with intensive malaria screening and prompt treatment," Malaria Journal, vol. 12, no. 1, article 139, 2013.

[4] E. Amuta, R. Houmsou, E. Wama, and M. Ameh, "Malarial infection among antenatal and maternity clinics attendees at the federal medical centre, Makurdi, Benue State, Nigeria," Infectious Diseases Reports, vol. 6, no. 1, p. 5050, 2014.

[5] A. Ouédraogo, A. B. Tiono, A. Diarra et al., "Transplacental transmission of Plasmodium falciparum in a highly malaria endemic area of Burkina Faso," Journal of Tropical Medicine, vol. 2012, Article ID 109705, 7 pages, 2012.

[6] M. C. Tahita, H. Tinto, J. Menten et al., "Clinical signs and symptoms cannot reliably predict Plasmodium falciparum malaria infection in pregnant women living in an area of high seasonal transmission," Malaria Journal, vol. 12, no. 1, article 464, 2013.

[7] C. J. Uneke, "Impact of placental Plasmodium falciparum malaria on pregnancy and perinatal outcome in sub-Saharan Africa. II: effects of placental malaria on perinatal outcome; malaria and HIV," Yale Journal of Biology and Medicine, vol. 80, no. 3, pp. 95-103, 2007.

[8] Z. Douamba, C. Bisseye, F. W. Djigma et al., "Asymptomatic malaria correlates with anaemia in pregnant women at Ouagadougou, Burkina Faso," Journal of Biomedicine and Biotechnology, vol. 2012, Article ID 198317, 6 pages, 2012.

[9] S. J. Rogerson, L. Hviid, P. E. Duffy, R. F. Leke, and D. W. Taylor, "Malaria in pregnancy: pathogenesis and immunity," The Lancet Infectious Diseases, vol. 7, no. 2, pp. 105-117, 2007.

[10] L. Barfod, T. Dobrilovic, P. Magistrado et al., "Chondroitin sulfate a-adhering Plasmodium falciparum-infected erythrocytes express functionally important antibody epitopes shared by multiple variants," The Journal of Immunology, vol. 185, no. 12, pp. 7553-7561, 2010.

[11] S. Chaikitgosiyakul, M. J. Rijken, A. Muehlenbachs et al., "A morphometric and histological study of placental malaria shows significant changes to villous architecture in both Plasmodium falciparum and Plasmodium vivax infection," Malaria Journal, vol. 13, no. 1, article 4, 2014.
[12] H. L. Guyatt and R. W. Snow, "Impact of malaria during pregnancy on low birth weight in sub-Saharan Africa," Clinical Microbiology Reviews, vol. 17, no. 4, pp. 760-769, 2004.

[13] M. Desai, F. O. ter Kuile, F. Nosten et al., "Epidemiology and burden of malaria in pregnancy," The Lancet Infectious Diseases, vol. 7, no. 2, pp. 93-104, 2007.

[14] R. W. Steketee, B. L. Nahlen, M. E. Parise, and C. Menendez, "The burden of malaria in pregnancy in malaria-endemic areas," American Journal of Tropical Medicine and Hygiene, vol. 64, no. 1-2, pp. 28-35, 2001.

[15] N. G. Schwarz, A. A. Adegnika, L. P. Breitling et al., "Placental malaria increases malaria risk in the first 30 months of life," Clinical Infectious Diseases, vol. 47, no. 8, pp. 1017-1025, 2008.

[16] S. Borgella, N. Fievet, B.-T. Huynh et al., "Impact of pregnancyassociated malaria on infant malaria infection in southern Benin," PLoS ONE, vol. 8, no. 11, Article ID e80624, 2013.

[17] I. Malhotra, A. Dent, P. Mungai et al., "Can prenatal malaria exposure produce an immune tolerant phenotype? A prospective birth cohort study in Kenya," PLoS Medicine, vol. 6, no. 7, Article ID e1000116, 2009.

[18] A. Rachas, A. Le Port, G. Cottrell et al., "Placental malaria is associated with increased risk of nonmalaria infection during the first 18 months of life in a beninese population," Clinical Infectious Diseases, vol. 55, no. 5, pp. 672-678, 2012.

[19] J. Ismaili, M. Van Der Sande, M. J. Holland et al., "Plasmodium falciparum infection of the placenta affects newborn immune responses," Clinical and Experimental Immunology, vol. 133, no. 3, pp. 414-421, 2003.

[20] C. Bisseye, M. van der Sande, W. D. Morgan, A. A. Holder, M. Pinder, and J. Ismaili, "Plasmodium falciparum infection of the placenta impacts on the T helper type 1 (Th1)/Th2 balance of neonatal $\mathrm{T}$ cells through $\mathrm{CD} 4{ }^{+} \mathrm{CD} 25^{+}$forkhead box $\mathrm{P}^{+}$ regulatory T cells and interleukin-10," Clinical \& Experimental Immunology, vol. 158, no. 3, pp. 287-293, 2009.

[21] M. S. Mackroth, I. Malhotra, P. Mungai, D. Koech, E. Muchiri, and C. L. King, "Human cord blood $\mathrm{CD} 4{ }^{+} \mathrm{CD} 25^{\text {hi }}$ regulatory T cells suppress prenatally acquired T cell responses to Plasmodium falciparum antigens," Journal of Immunology, vol. 186, no. 5, pp. 2780-2791, 2011.

[22] A. Moody, "Rapid diagnostic tests for malaria parasites," Clinical Microbiology Reviews, vol. 15, no. 1, pp. 66-78, 2002.

[23] S. Gies, S. O. Coulibaly, F. T. Ouattara, and U. D'Alessandro, "Individual efficacy of intermittent preventive treatment with sulfadoxine-pyrimethamine in primi- and secundigravidae in rural Burkina Faso: impact on parasitaemia, anaemia and birth weight," Tropical Medicine \& International Health, vol. 14, no. 2, pp. 174-182, 2009.

[24] T. P. Eisele, D. A. Larsen, P. A. Anglewicz et al., "Malaria prevention in pregnancy, birthweight, and neonatal mortality: a meta-analysis of 32 national cross-sectional datasets in Africa," The Lancet Infectious Diseases, vol. 12, no. 12, pp. 942-949, 2012.

[25] S. Bamba, A. Séré, R. Nikiéma et al., "Intermittent preventive treatment with sulfadoxine-pyrimethamine for malaria in pregnant women: efficacy and compliance in two urban hospitals in Burkina Faso," The Pan African Medical Journal, vol. 14, article 105, 2013.

[26] B. J. Brabin, B. F. Kalanda, F. H. Verhoeff, L. H. Chimsuku, and R. L. Broadhead, "Risk factors for fetal anaemia in a malarious area of Malawi," Annals of Tropical Paediatrics, vol. 24, no. 4, pp. 311-321, 2004. 
[27] A. K. Laar, F. E. Grant, Y. Addo et al., "Predictors of fetal anemia and cord blood malaria parasitemia among newborns of HIVpositive mothers," BMC Research Notes, vol. 6, no. 1, article 350, 2013.

[28] A. K. Onyebuchi, L. O. Lawani, C. A. Iyoke, C. R. Onoh, and N. E. Okeke, "Adherence to intermittent preventive treatment for malaria with sulphadoxine-pyrimethamine and outcome of pregnancy among parturients in South East Nigeria," Patient Preference and Adherence, vol. 8, pp. 447-452, 2014.

[29] F. E. A. Lesi, M. Y. Mukhtar, E. U. Iroha, and M. T. C. EgriOkwaji, "Clinical presentation of congenital malaria at the Lagos University Teaching Hospital," Nigerian Journal of Clinical Practice, vol. 13, no. 2, pp. 134-138, 2010.

[30] I. C. J. Omalu, C. Mgbemena, A. Mgbemena et al., "Prevalence of congenital malaria in Minna, North Central Nigeria," Journal of Tropical Medicine, vol. 2012, Article ID 274142, 5 pages, 2012.

[31] B. U. Oraneli, O. C. Okeke, and P. O. Ubachukwu, "Effect of placental malaria on birth weight of babies in Nnewi, Anambra state, Nigeria," Journal of Vector Borne Diseases, vol. 50, no. 1, pp. 13-17, 2013.

[32] A. Gnémé, W. M. Guelbéogo, M. M. Riehle et al., “Plasmodium species occurrence, temporal distribution and interaction in a child-aged population in rural Burkina Faso," Malaria Journal, vol. 12, no. 1, article 67, 2013.

[33] C. O. Agomo and W. A. Oyibo, "Factors associated with risk of malaria infection among pregnant women in Lagos, Nigeria," Infectious Diseases of Poverty, vol. 2, article 19, 2013.

[34] C. Roucher, C. Rogier, C. Sokhna, A. Tall, and J. F. Trape, "A 20year longitudinal study of Plasmodium ovale and Plasmodium malariae prevalence and morbidity in a West African population," PLoS ONE, vol. 9, no. 2, Article ID e87169, 2014.

[35] J. Singh, D. Soni, D. Mishra, H. P. Singh, and S. Bijesh, "Placental and neonatal outcome in maternal malaria," Indian Pediatrics, vol. 51, no. 4, pp. 285-288, 2014.

[36] L. Kalilani-Phiri, P. C. Thesing, O. M. Nyirenda et al., "Timing of malaria infection during pregnancy has characteristic maternal, infant and placental outcomes," PLoS ONE, vol. 8, no. 9, Article ID e74643, 2013. 


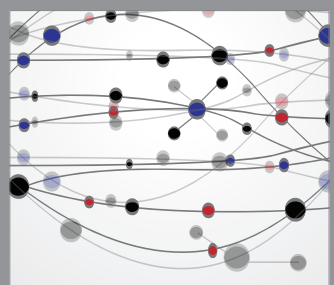

The Scientific World Journal
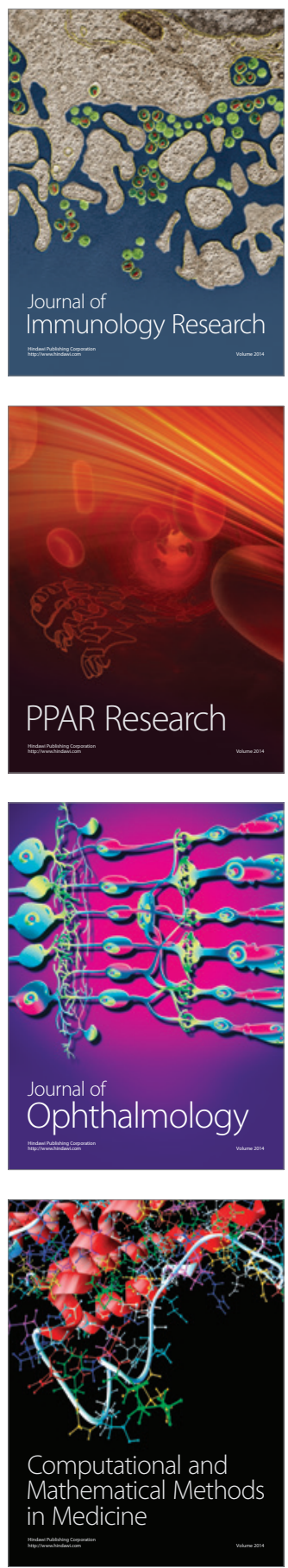

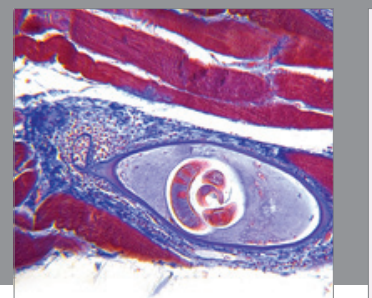

Gastroenterology

Research and Practice
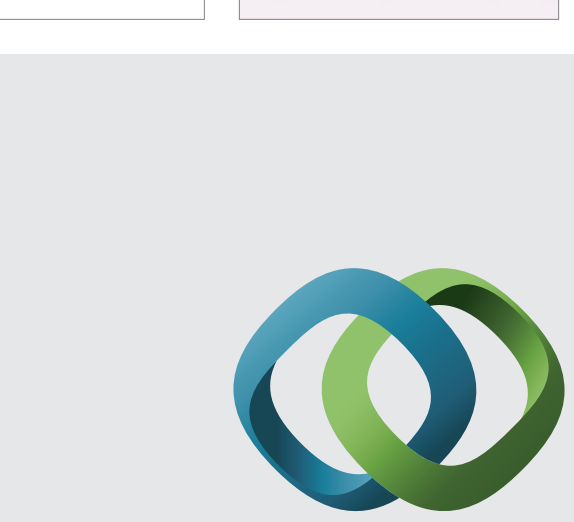

\section{Hindawi}

Submit your manuscripts at

http://www.hindawi.com
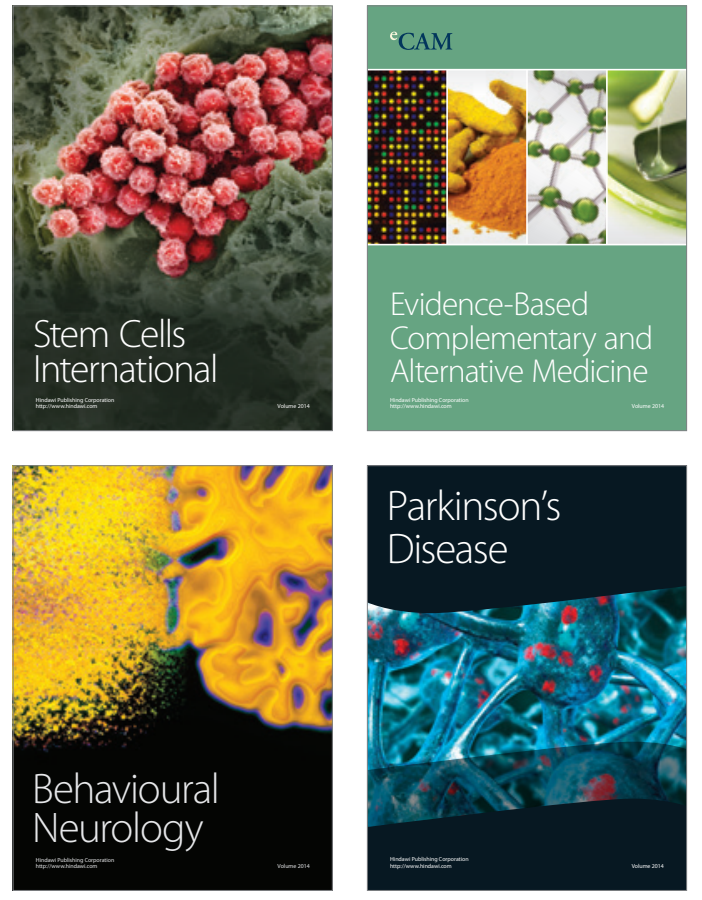
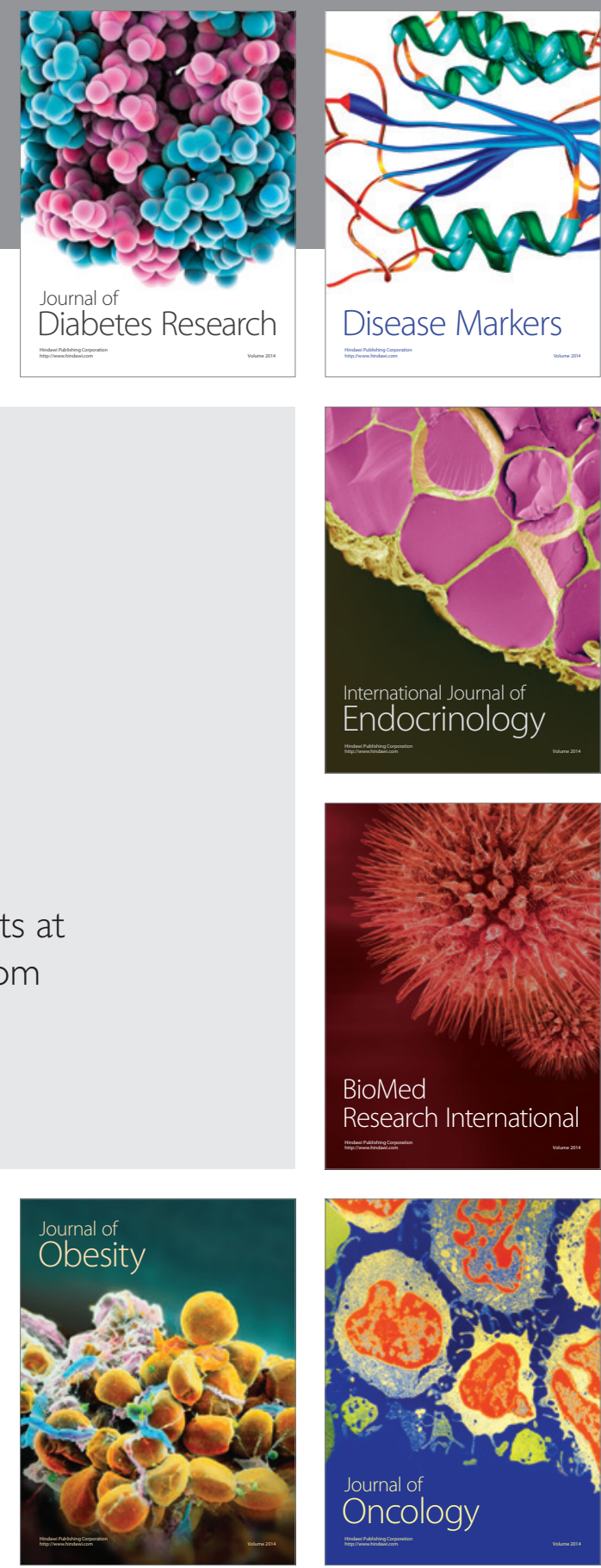

Disease Markers
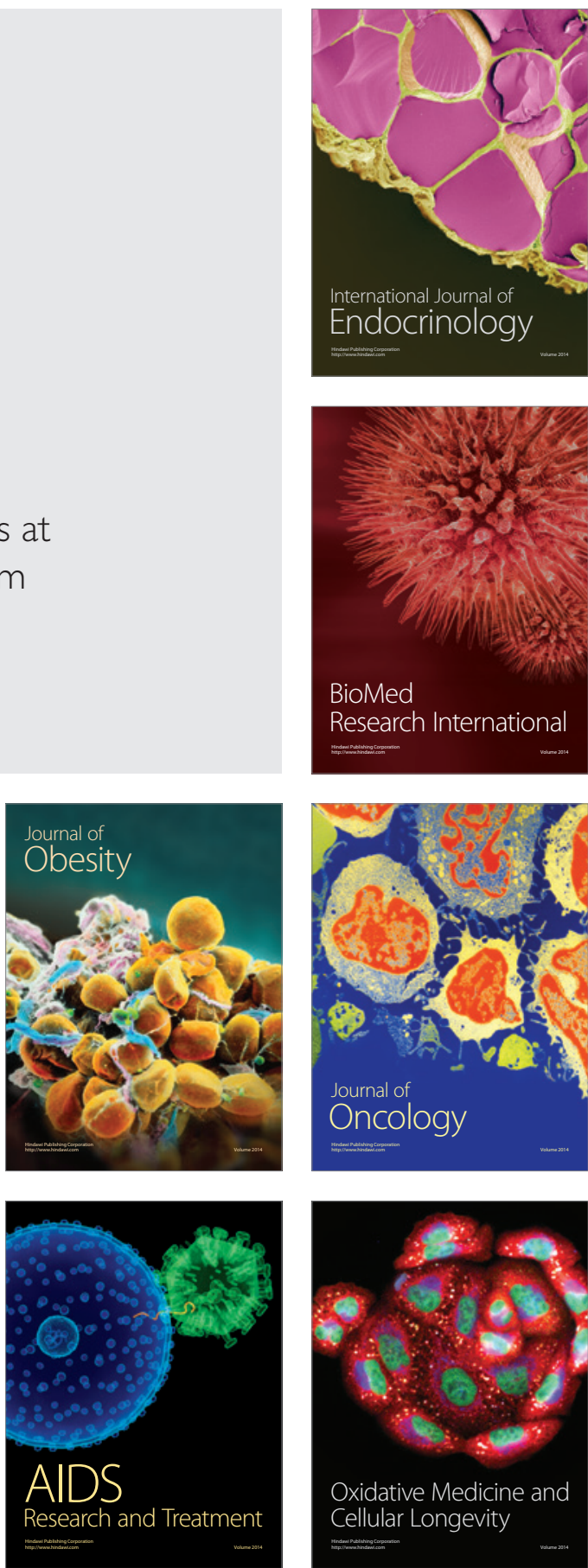